SECTION 31. Economic research, finance, innovation, risk management.

Anatoly Aleksandrovich Naumov Docent, Candidate of Technical Sciences, Center of Applied Mathematical Research, Novosibirsk, Russia a_a_naumov@mail.ru

Evgeniy Borisovich Uvarov General Director, Microclimate Service, Moscow, Russia rapid1@,mail.ru

\title{
FINANCIAL LEVERAGE: PROBLEMS AND SOLUTIONS
}

Abstract: In this paper analysis of some models and methods of financial leverage theory given. Incorrectness of models is shown. Approaches to remedy them are offered.

Keywords: Financial leverage, financial analysis, capital structure, borrowings, optimization, simulation, financial flows, the method of detail flows.

Citation: Naumov AA, Uvarov EB (2014) FINANCIAL LEVERAGE: PROBLEMS AND SOLUTIONS. ISJ Theoretical \& Applied Science 9 (17): 180-182. doi: http://dx.doi.org/10.15863/TAS.2014.09.17.31

\section{УДК 336.77: 330.42}

\section{ФИНАНСОВЫЙ РЫЧАГ: ПРОБЛЕМЫ И РЕШЕНИЯ}

Аннотация: В работе приведены результаты анализа некоторых моделей $и$ методов теории финансового рычага. Показаны некорректности моделей. Предложены подходы по их исправлению.

Ключевые слова: Финансовый рычаг, финансовый анализ, структура капитала, заимствования, оптимизация, моделирование, финансовые потоки, метод детализации потоков.

Введение. Написанию настоящей работы способствовало появление публикаций [1], [2]. Финансовый рычаг - интересная с научной и прикладной точек зрения тема. Однако, некоторые публикации в этой области (в частности, [1]) не лишены принципиальных ошибок, которые не позволяют идеями этих работ эффективно воспользоваться на практике. Заметим, что некоторые решения задачи построения эффективного финансового рычага, пусть и не для самых общих случаев, предложены в работах [3] - [5]. В этой работе мы рассмотрим типичные ошибки, которые возникают при рассмотрении вопросов заимствования и построения на их основе финансовых рычагов, и пути их возможного устранения.

Проблемы одной модели финансового рычага. Рассмотрим проблемы модели для нахождения оптимальных значений параметров финансового рычага, рассмотренной в работе [1]. Так, в этой работе эффективность от финансового рычага предлагается оценивать в соответствии с критерием рентабельности собственного капитала: $R_{\text {СК }}=\left(R_{\text {пр }}+\left(R_{\text {пр }}-r\right) \cdot \frac{3 K}{C K}\right) \cdot(1-N)$, где $R_{\text {Ск }}-$ рентабельность собственного капитала $(C K) ; R_{\text {пр }}$ - производственная рентабельность; $r$ - стоимость привлечения заемного капитала (3K); $N$ - налог на прибыль. С помощью несложных преобразований это выражение для рентабельности можно привести к виду: $R_{\mathrm{C \kappa}}=\frac{\left(\Pi_{э к}-r \cdot 3 K\right) \cdot(1-N)}{C K}$, где 
$\Pi_{\text {эк }}=R_{\text {пр }} \cdot(C K+3 K)-$ экономическая прибыль. Конечно, в таком виде критерий рентабельности выглядит более понятным: в числителе дроби представлена прибыль после расчета за заимствования $(r \cdot 3 K)$ и выплаты налогов.

Замечания. Во-первых, учитываются выплаты только процентов за использование $3 K$. Во-вторых, в бизнесе будет задействован капитал в размере $(C K+3 K)$ от двух источников финансирования, а рентабельность оценивается только относительно собственных средств. В-третьих, в этих формулах предполагается, что эффект от финансового рычага будет оценен только на предстоящий период (т.е. только на один временной такт вперед).

К погашению заемных средств. В работе [1] предлагается учитывать поток погашений по заемным средствам в соответствии $\mathrm{c}$ формальной записью: $\Pi_{\mathrm{q}_{1}}=\left(\Pi_{\text {эк }}-q_{0} \cdot 3 K_{0}-q_{1} \cdot \Delta 3 K_{1}\right) \cdot(1-N)$. Здесь $\Pi_{\mathrm{q}_{1}}-$ чистая прибыль бизнеса в прогнозном периоде (индекс «1»), П эк - экономическая прибыль в прогнозном периоде, $R_{\mathrm{3K}_{0}}=q_{0} \cdot 3 K_{0} \quad$ и $R_{\Delta 3 \mathrm{~K}_{1}}=q_{1} \cdot \Delta 3 K_{1}$ - платежи по заемным средствам $3 K_{0}$ и $\triangle 3 K_{1}$ в отчетном (индекс «0») и прогнозном периодах соответственно, $q_{0}$ и $q_{1}$ - коэффициенты, определяющие величины этих платежей. Как было отмечено выше, здесь сделана попытка ввести в рассмотрение потоки платежей за заимствование (на два временных такта), но сделано это неаккуратно. Понятно, что прибыль $\Pi_{\text {эк }}$ будет получена в конце прогнозируемого периода и в соответствии с этой формулой погашения по кредитам (в том числе и $R_{3 \mathrm{~K}_{0}}=q_{0} \cdot 3 K_{0}$ ) предлагается произвести именно в это время. Но, это можно было (и нужно было) делать в отчетном периоде из прибыли $\Pi_{\text {эк }}$. В противном случае, если с учетом сделанного замечания не произвести изменения в расчетной формуле для $\Pi_{\mathrm{u}_{1}}$, то этой формулой нельзя будет воспользоваться для числа временных периодов большем, чем два.

Собственный капитал. Предположим, что общий объем капитала $C K$ решено

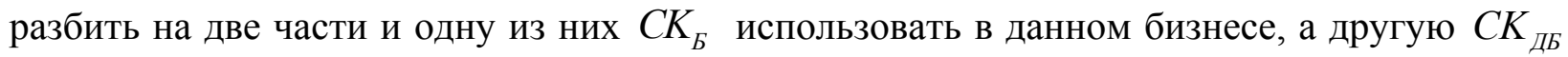
использовать вне его, в другом, альтернативном бизнесе (конечно, выполняется $\left.C K=C K_{b}+C K_{д ь}\right)$. Тогда это обстоятельство должно найти отражение в формуле для расчета эффективности рычага. Например, это можно представить следующим образом: $R_{\mathrm{K}_{o \sigma}}=\frac{\left(\Pi_{э к}+(1+i) \cdot C K_{д Б}-(1+r) \cdot 3 K\right) \cdot(1-N)}{C K_{Б}+3 K}$, где $i-$ доходность альтернативного бизнеса.

Как должна решаться (и решается) задача использования критерия финансового рычага на практике.

1. Необходимо построить прогноз для общего объема требуемого капитала на прогнозный период $\left(K_{\text {об }}=C K+3 K\right)$;

2. Следует оценить размер $C K$, который будет доступен для вложения в бизнес на предстоящий период;

3. Определить объем заимствования по формуле: $3 K=K_{\text {об }}-C K$ и в соответствии с критерием (доход, доходность, рентабельность и пр.) оценить эффективность вариантов заимствования (сроки, ставки, объемы заимствований и т.д.); решить задачу перебора вариантов (параметров) заимствования $3 \mathrm{~K}$;

4. Выбрать лучшую из рассмотренных схем заимствования. 
Прогнозируемые значения величин $K_{\text {об }}, r$ и других могут содержать ошибки, в этом случае следует оценивать как значения критериев эффективности, так и значения возникающих при этих ошибках рисков (см. подробнее в [7], [9], [10]).

\section{Выводы.}

1. Моделями финансового рычага (например, такими, какие были предложены в работе [1]) следует пользоваться с определенной степенью осторожности. Они могут не отражать прикладную суть задач оптимизации схем заимствования капитала и приводить к ложным рекомендациям по поводу развития бизнеса.

2. В общем виде задача использования финансового рычага должна опираться на динамическую модель финансовых потоков (входных, выходных, заимствований, внешнего использования и пр.) и некоторые варианты таких моделей представлены в работах [4], [7].

3. К сожалению, «латание дыр» для моделей финансового рычага в виде незначительных их корректировок (см. замечания и правки моделей выше) не приведет к существенному их улучшению и потребуется их кардинальное осмысление и изменение. Причем, основой для таких изменений могут послужить потоковые модели бизнеспроцессов и использование методов детализации финансовых потоков (см. [6], [7], [10]).

\section{References:}

1. Kirillov YuV, Nazimko EN (2014) Ekonomiko-matematicheskiy analiz effekta finansovogo ryichaga. Finansovaya analitika: problemyi i resheniya, No. 34, pp. 56 - 63.

2. Salmin PS, Salmina NA (2014) Parametricheskoe modelirovanie effekta finansovogo ryichaga. Ekonomicheskiy analiz: teoriya i praktika, No. 27, pp. $27-34$.

3. Naumov AA (2013) K analiticheskim resheniyam nekotoryih ekonomiko-matematicheskih zadach. Materials of the ISPC «Results\&Perspectives», 30.09.2013, Florence, Italy. Theoretical \& Applied Science 9 (5): 93-97. http://dx.doi.org/10.15863/TAS.2013.09.5.11

4. Naumov AA (2013) Optimizatsiya strukturyi zaimstvovaniy i vlozheniy dohodov investitsionnogo proekta. Materials of the ISPC «Advances in techniques\&technologies», 30.10.2013, Milan, Italy. Theoretical \& Applied Science 10(6): 133-136. doi: http://dx.doi.org/10.15863/TAS.2013.10.6.20

5. Naumov AA (2013) K voprosu ob uproschenii dvuh zadach optimizatsii investitsiy. Finansovaya analitika: problemyi i resheniya, No. 46 (184), pp. 26 - 30.

6. Naumov AA (2013) Ispolzovanie metoda detalizatsii finansovyih potokov $\mathrm{v}$ zadachah otsenivaniya effektivnosti proektov. Finansovaya analitika: problemyi i resheniya, No. 48 (186), pp. $35-41$.

7. Naumov AA (2013) Metodyi analiza i sinteza investitsionnyih proektov. Effektivnost, riski, upravlenie. LAP LAMBERT Academic Publishing: 356.

8. Naumov AA (2014) Otsenivanie effektivnosti integrirovannyih proektov. Finansovaya analitika: problemyi i resheniya, No. 8 (194), pp. 36 - 43.

9. Naumov AA (2014) Analiz kriteriev effektivnosti investitsionnyih proektov. Materials of the ISPC «Modern mathematics in science», 30.06.2014, Caracas, Venezuela. ISJ $\begin{array}{llllll}\text { Theoretical\&Applied } & \text { Science } & 6 & \text { (14): } & 92-94 . & \text { doi: }\end{array}$ http://dx.doi.org/10.15863/TAS.2014.06.14.19

10. Spisok trudov (2014) Available: https://sites.google.com/site/anatolynaumov2011/home/spisok-trudov-list-of-papers Accessed: 2014 Sep 25. 